\title{
Abstract
}

\section{Traumatic vitreous haemorrhage in adults: A two year experience}

Ekanayake E M S $B^{1}$, Samarasinghe $G P^{1}$, Sriharanathan $P^{1}$

${ }^{1}$ Teaching Hospital, Kandy, Sri Lanka

Key words: vitreous haemorrhage, traumatic

\begin{abstract}
Introduction
Vitreous haemorrhage $(\mathrm{VH})$ is the presence of extravasated blood within the vitreous cavity. Traumatic injury contributes to significant ocular morbidity in our settings.

Objective

To ascertain the pattern/s of injury in traumatic $\mathrm{VH}$ in adult patients who attended the Eye unit, TH Kandy.

Methods

Retrospective analysis of adults with traumatic VH between September 2013 and August 2015

Results

During the study period 58(24.5\%) adults presented with traumatic VH out of 236 adults presented with a vitreous haemorrhage. Out of these, $86 \%$ were males. The mean age was 43 years (20-72 years). The majority were at or below 40 years $(41.4 \%)$.

Most of them had sudden loss of vision (77\%) and the right eye was involved in most instances (69\%). Presenting visual acuity ranged from 6/36 to non perception of light (NPL). Mean intraocular pressure was $16 \mathrm{mmHg}$ (6-30). All the patients were free of co-morbidities except one patient who had hypertension.

Trauma profile accounted for; blunt trauma (67\%), penetrating injury $(21 \%)$ and blast injuries (12\%).

Associated ocular injuries were corneal laceration (69\%), iris damage (20\%), lens subluxation (36\%) and retinal detachment (27.5\%)

Conclusion

Nearly a quarter of VHs occur due to trauma which has a highest incidence among the young adults. Blunt or penetrating trauma can injure intact vessels directly therefore it is the leading cause of $\mathrm{VH}$ in young adults and it is a preventable etiology
\end{abstract}

Corresponding Author: EMSB Ekanayake, Email:<sb81ekanayake@gmail.com>

Presentation at the $5^{\text {th }}$ biennial academic sessions of the Postgraduate institute of Medicine on 06 October 2016

Competing Interests: Authors have declared that no competing interests exist. 